healthcare professionals. Likewise, doctors are uncertain about dental neglect. Minimal joint standards for dental neglect thresholds and the appropriate response need to be agreed.

\section{G423 SERVICE EVALUATION OF THE MANAGEMENT OF OSTEOARTICULAR INFECTION OVER 8 YEARS IN A SINGLE CENTRE}

${ }^{1}$ A Rodrigues Da Costa, ${ }^{1} \mathrm{~B}$ Oguti, ${ }^{1} \mathrm{~A}$ Ashby, ${ }^{2} \mathrm{~A}$ Smith, ${ }^{3} \mathrm{E}$ Lim, ${ }^{4} \mathrm{E}$ Alexander, ${ }^{5} \mathrm{~K}$ Fidler. ${ }^{1}$ Paediatrics, Royal Alexandra Hospital, Brighton and Sussex University Hospitals, Brighton, UK; ${ }^{2}$ Orthopaedics, Royal Alexandra Hospital, Brighton and Sussex University Hospitals, Brighton, UK; ${ }^{3}$ Paediatrics, Great North Childrens Hospital, Newcastle-UponTyne, UK; ${ }^{4}$ Microbiology, Royal Alexandra Hospital, Brighton and Sussex University Hospitals, Brighton, UK; ${ }^{5}$ Paediatrics, Brighton and Sussex Medical School, Brighton, UK

\subsection{6/archdischild-2015-308599.377}

Aims There is no national consensus on the management of osteoarticular infection (OAI). Practice varies widely throughout the United Kingdom, with differences in duration of antibiotic treatment, switching from intravenous (IV) to oral antibiotics and requirement for peripherally inserted central venous catheters (PICC). Data about recurrence and complication rates is scant. We aimed to evaluate local practice: analyse demographic data, antibiotic course duration and administration route, use of invasive lines and associated complications with a view to guideline development. Methods OAI in children aged 0-17 years, presenting to one centre, from 01/09/2006-01/09/2014, identified through hospital coding, clinician and microbiology records.

Results Demographic Data

82 confirmed OAI cases: 55\% male, $45 \%$ female, mean age 4.8 years. Preliminary data from the first 24 cases revealed none had significant co-morbidities, sickle cell disease or immunosuppression.

Pathogenesis

Tibia most commonly affected site, followed by femur. Organisms isolated in under half the cases. 2 cases of severe PVL-MSSA disease identified

Management

Median duration of antibiotic course (IV and Oral) was 45 (range 7-358) days. Median duration of IV antibiotic course was 20 (range 6-75) days. Oral switch occurred in 76\%, after a median of 18 (range 3-17 days) of IV antibiotics. Most had PICC lines inserted, complications included line sepsis (x1). Complication of OAI included hyperesthesia, and prolonged chronic OAI

Conclusions The first 24/82 cases analysed confirm wide variation in management, partly due to the diversity in age, presentation and organism. This highlights the difficulties for guideline development for this heterogeneous group. Full analysis will be presented at the conference. It will provide a comprehensive picture of current local practice, and add to national data being collected as part of the DINOSAUR study, to further understanding of this serious condition.

\section{G424 PREMATURITY AND THE BURDEN OF ASSESSMENT ON AUTISTIC SPECTRUM DISORDER DIAGNOSTIC SERVICES: A PRAGMATIC APPROACH}

R Sharma, H Gillet. Paediatric Department, Great Western Hospital, Swindon, UK

10.1136/archdischild-2015-308599.378
Aim Prematurity is a risk factor for autism with prevalence in middle childhood increasing $2-4$ fold in preterm versus term populations ${ }^{1,2,3,4}$ with a dose-response relationship between gestation and adjusted HR for autism. ${ }^{1} 8 \%$ of the EPICure cohort screened positive for ASD at 11 years. ${ }^{2}$ There are no pragmatic UK studies examining the burden of the preterm population on ASD assessment services. The aim of this abstract is to determine the burden of autistic spectrum disorder assessments in a cohort of extreme preterm versus moderately preterm versus term children.

Methods Retrospective cohort analysis of all preterm deliveries less than 36 weeks gestation from January to December 2009 that survived to discharge. Data gathered from local health records on gestation and gender then cross referenced with local health records to determine whether ASD assessment undertaken. Those with HIE, neurometabolic conditions, congenital cardiac malformations or chromosomal disorders were excluded. Results 198 preterm infants were included. 60 cases in the extreme preterm group. $8.3 \%$ of this population underwent ASD assessment by 5 years of age. 138 cases in the moderate preterm group. $4.3 \%$ of this population underwent assessment. In the general term population 4202 children were born in 2009. Only 2\% of these were assessed for autism.

Conclusion Extreme preterm infants are approximately four times more likely to undergo diagnostic assessment for ASD than the term population. Moderately preterm populations are approximately twice as likely to undergo assessment. Recognition of the burden of these high risk groups on ASD assessment services may aid in service provision planning.

\section{REFERENCES}

1 Kuzniewicz MW. Prevalence and neonatal factors associated with autism spectrum disorders in preterm infants. J Pediatr. 2014;164:20-5

2 Johnson $\mathrm{S}$, Hollis $\mathrm{C}$, Kochhar $\mathrm{P}$, et al. Psychiatric disorders in extremely preterm children: longitudinal finding at age 11 years in the EPICure study. I Am Acad Child Adolesc Psychiatry 2010;49:453-63

3 Hwang $Y-S$, Weng S-F, Cho C-Y, Tsai W-H. Higher prevalence of autism in Taiwanese children born prematurely: A nationwide population-based study. Res Dev Disabil. 2013;34:2462-2468

4 Woodward LJ, Bora S, Moor S. Psychiatric outcomes at age 9 of a regional cohort of children born very preterm. J Paediatr Child Health 2012;48: $1034-4810$

\section{G425 ROTAVIRUS VIREMIA AND GENOTYPE CHARACTERISATION AMONG CHILDREN WITH ROTAVIRUS DIARRHOEA PRESENTING TO A TEACHING HOSPITAL}

${ }^{1} \mathrm{~S}$ Kwarteng Owusu, ${ }^{2} \mathrm{D}$ Ansong, ${ }^{2} \mathrm{CKA}$ Poku, ${ }^{1}$ RKK Owusu, ${ }^{1} \mathrm{MO}$ Owusu, ${ }^{2} \mathrm{E}$ Addo-Yobo, ${ }^{3} \mathrm{G}$ Armah, ${ }^{4} \mathrm{~K}$ Ampofo. ${ }^{1}$ Child Health, Komfo Anokye Teaching Hospital, Kumasi, Ghana; ${ }^{2}$ Child Health, Kwame Nkrumah University of Science and Technology-School of Medical Scienc, Kumasi, Ghana; ${ }^{3}$ Department of Virology, Nougouchi Memorial Institute for Research, Accra, Ghana; ${ }^{4}$ Department of Paediatrics, University of Utah, Salt Lake City, United States of America

\subsection{6/archdischild-2015-308599.379}

Introduction Rotavirus (RV) is the leading cause of diarrhoea in children $<5$ years worldwide, especially in developing countries. $\mathrm{RV}$ viremia has been detected in some children with RV diarrhoea, especially during early RV infection and severe disease. There is however a paucity of data on the genotypes associated with viremia during acute RV diarrhoea. This study evaluated the burden of RV viremia among children with RV diarrhoea and circulating genotypes in blood and stools. 